\title{
Bouncing ball problem: Stability of the periodic modes
}

\author{
Joaquim J. Barroso \\ Associated Plasma Laboratory, National Institute for Space Research, \\ 12227-010, São José dos Campos, SP, Brazil \\ Marcus V. Carneiro and Elbert E. N. Macau \\ Computing and Applied Mathematics Laboratory, National Institute for Space Research, \\ 12227-010, São José dos Campos, SP, Brazil
}

(Received 7 October 2008; published 6 February 2009)

\begin{abstract}
Exploring all its ramifications, we give an overview of the simple yet fundamental bouncing ball problem, which consists of a ball bouncing vertically on a sinusoidally vibrating table under the action of gravity. The dynamics is modeled on the basis of a discrete map of difference equations, which numerically solved fully reveals a rich variety of nonlinear behaviors, encompassing irregular nonperiodic orbits, subharmonic and chaotic motions, chattering mechanisms, and also unbounded nonperiodic orbits. For periodic motions, the corresponding conditions for stability and bifurcation are determined from analytical considerations of a reduced map. Through numerical examples, it is shown that a slight change in the initial conditions makes the ball motion switch from periodic to chaotic orbits bounded by a velocity strip $v= \pm \Gamma /(1-\varepsilon)$, where $\Gamma$ is the nondimensionalized shaking acceleration and $\varepsilon$ the coefficient of restitution which quantifies the amount of energy lost in the ball-table collision.
\end{abstract}

DOI: 10.1103/PhysRevE.79.026206

PACS number(s): 05.45.-a, 81.05.Rm

\section{INTRODUCTION}

One of the simplest dynamical systems displaying a rich variety of nonlinear behavior is the bouncing ball model. Consisting of a ball bouncing vertically under the action of gravity on a massive sinusoidally vibrating platform, such a deterministic system exhibits large families of irregular nonperiodic solutions and fully developed chaos in addition to harmonic and subharmonic motions [1] depending upon the amplitude and frequency of the driving platform and also on the coefficient of restituition, $0<\varepsilon<1$, which accounts for the amount of energy dissipated during each collision, with the elastic limit $\varepsilon=1$ giving rise to unbounded nonperiodic or stochastic motions [2].

Simple yet fundamental, this system is naturally connected to physical and engineering problems ranging from nanotechnology to astrophysics. In fact, the bouncing ball system has been used as a simplified model of vibrated granular materials which can be regarded as an assembly of bouncing balls, each one colliding inelastically with the walls of the container and the adjacent balls [3]. Also the bouncing ball problem is closely related to Fermi acceleration, a model pictured by Fermi to explain the extra solar origin and the buildup of energy of cosmic rays, whereby the cosmic-ray particles are accelerated in interstellar space by collisions against wandering magnetic fields which occupy the interstellar medium [4,5]. Moreover, the bouncing ball model is shown to be relevant to nonlinear systems subject to periodic excitation, such as moored ships driven by steady ocean waves [6] or materials in mechanical contact on a nanometer scale, as in atomic-force-microscopic devices in which the tip of the cantilever acts as the bouncing mass [7].

In past studies, the bouncing ball problem has been examined in many of its ramifications. The first systematic study is attributed to Holmes [8], upon constructing a discrete map whereby the conditions for stability and bifurcation of peri- odic trajectories are determined on the assumption that the jumps of the ball are larger compared to the overall displacement of the table. Other studies based on the differential equation of motion of the ball [9], or else using a mapping approach similar to Holmes's, investigated chaotic response and manifold collisions [10], period-doubling regime [11], noise-induced chaotic motion [12], the completely inelastic case [13], rate of energy input into the system [14,15], and chattering mechanisms through which the ball gets locked on the vibrating table [16].

Despite these earlier conceptual studies, there still remains a lack of information on how to access the appropriate driving parameters (namely, the frequency and the excitation amplitude) and also the starting conditions so as to drive the ball into a prescribed oscillation mode at a given coefficient of restitution. To supplement this kind of information and extend past studies, the present paper embraces analytical methods accompanied by computer simulation to examine in a unified way the rich phase behavior of the bouncing ball with emphasis on the driving and launching parameters so that the ball dropped at zero initial velocity might evolve to a desired periodic orbit and keep bouncing there. Additional information focuses on how the bouncing ball dynamics is susceptible to changes in the starting conditions.

\section{DYNAMICAL EQUATIONS AND MODE STABILITY}

This section gives a mathematical description of an elastic ball, with coefficient of restitution $\varepsilon$, which is kept continually bouncing off a vertically oscillating base. Infinitely massive, the platform is fixed to a rigid frame which vibrates sinusoidally as $S(t)=A \sin \omega t$ so as to maintain the motion of the ball, whose dynamics is governed by a gravitational field $g$ and the impacts with the base (Fig. 1). The next collision time after the departure time $t_{i}$ from the platform is the smallest solution $t_{i+1}>t_{i}$ of the discrete-time dynamics map 


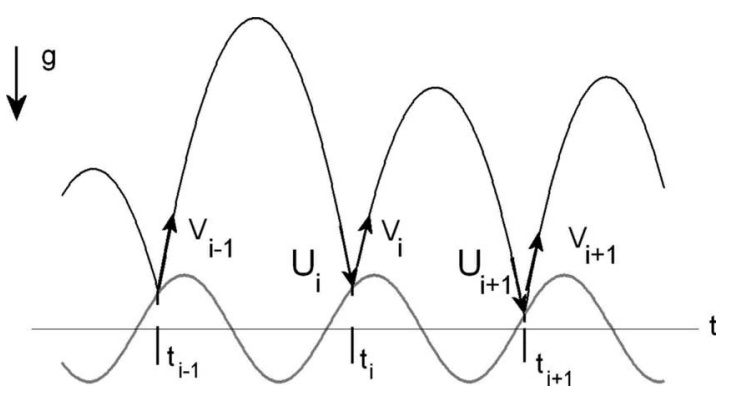

FIG. 1. One-dimensional ball bouncing off a sinusoidally vibrating table. The table and ball trajectories are depicted by solid lines over time.

$$
A\left(\sin \omega t_{i+1}-\sin \omega t_{i}\right)=V_{i}\left(t_{i+1}-t_{i}\right)-\frac{1}{2} g\left(t_{i+1}-t_{i}\right)^{2},
$$

where $V_{i}$ is the post-impact velocity (Fig. 1), which relates to the pre-impact $U_{i+1}$ velocity at time $t_{i+1}$ through

$$
U_{i+1}=V_{i}-g\left(t_{i+1}-t_{i}\right) .
$$

As far as the collision is partially elastic, the ball bounces back instantanteously at $t_{i+1}$ with a relative positive velocity

$$
V_{i+1}-\dot{S}\left(t_{i+1}\right)=-\varepsilon\left[U_{i+1}-\dot{S}\left(t_{t+1}\right)\right],
$$

where the relative landing velocity $U_{i+1}-\dot{S}\left(t_{t+1}\right)$ is always negative. Physically, the coefficient $\varepsilon$ (defined as the ratio of the relative velocities before and after the collision) gives a measure through the quantity $\left(1-\varepsilon^{2}\right)$ of the energy lost in the collision. Combining Eqs. (1)-(3) and nondimensionalizing the time and velocity variables according to $t_{i} \rightarrow \omega t_{i} \equiv \phi_{i}$ and $v_{i} \rightarrow V_{i} \omega / g$ gives the phase and velocity maps

$$
\begin{gathered}
\phi_{i+1}=\phi_{i}+\tau_{i}, \\
\Gamma\left[\sin \left(\phi_{i}+\tau_{i}\right)-\sin \left(\phi_{i}\right)\right]=v_{i} \tau_{i}-\frac{1}{2} \tau_{i}^{2}, \\
v_{i+1}=-\varepsilon\left(v_{i}-\tau_{i}\right)+(1+\varepsilon) \Gamma \cos \left(\phi_{i}+\tau_{i}\right),
\end{gathered}
$$

where $\Gamma=A \omega^{2} / g$ is the dimensionless shaking acceleration. With the state characterized by the phase $\phi_{i}$ and the postvelocity $v_{i}$, the above discrete map describes the complete bouncing ball dynamics, which is controlled by two parameters: namely, $\Gamma$ and $\varepsilon$.

Assuming that the height the ball reaches under ballistic flight is large compared with the table displacement, the interimpact time is well approximated by $\tau_{i}=2 v_{i}$, and therefore the system (5) reduces to the discrete dynamical system of the Zaslavski mapping [2]:

$$
\begin{aligned}
& T: \phi_{i+1}=\phi_{i}+2 v_{i} \bmod (2 \pi), \\
& T: v_{i+1}=\varepsilon v_{i}+(1+\varepsilon) \Gamma \cos \left(\phi_{i}+2 v_{i}\right),
\end{aligned}
$$

which can be iterated numerically upon starting from arbitrary initial conditions $\phi_{i}$ and $v_{i}$ to calculate the states of the forward $(i+1)$ or backward $(i-1)$ bounces. We note that the system (5) is invariant under phase displacement $\phi \rightarrow \phi$ $+2 \pi n, n= \pm 1, \pm 2, \ldots$, indicating that the phase space $(\phi, v)$

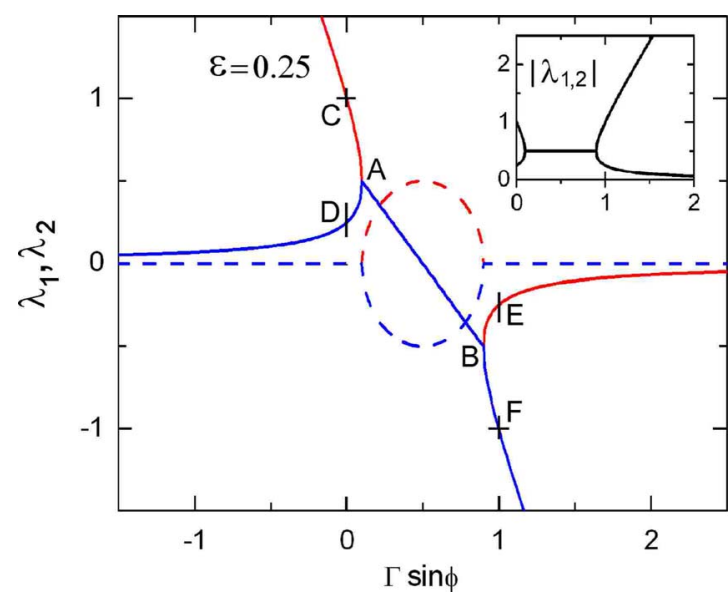

FIG. 2. (Color online) Domains of the eigenvalues of the system (5) at $\varepsilon=0.25$. Solid and dashed lines are for the real and imaginary parts of the eigenvalues, respectively. Negative values of $\sin \phi$ lead to instability from the left of point $C$ at which the system is in neutral equilibrium $\left(\lambda_{1}=+1\right)$. Increasing positive values of $\sin \phi$ from the origin first allow nodal (branch $C A D$ ) and then focal (segment $A B$ ) stability. Further increase of $\sin \phi$ from point $B$ results in nodal stability (branch $E B F$ ) turning into saddle-point stability at $F$ $\left(\lambda_{2}=-1\right)$. The stability domain with both eigenvalues $\left|\lambda_{1}, \lambda_{2}\right|<1$, corresponding to $0<\Gamma \sin \phi<1$, is shown in the inset.

can be obtained onto a cylinder by taking $\phi \bmod (2 \pi)$. Yet obtained in the context of the high-bounce approximation, here we note that the system (5) is exact for periodic orbits, while making identical periodic jumps the ball collides with the table at the same phase-i.e., $\sin \left(\phi_{i}+\tau_{i}\right)=\sin \phi_{i}$-and Eq. (5b) reduces to but a single expression $\tau_{i}=2 v_{i}$, otherwise obtained by invoking the high-bounce approximation.

In the following, we discuss the fact that the map $T$ exhibits a family of periodic orbits. To this end, we seek the fixed points of $T$ identified by $\left(\phi_{i+n}, v_{i+n}\right)=T^{n}\left(\phi_{i}, v_{i}\right)$ $=\left(\phi_{i}, v_{i}\right)$ such that $v_{n}=n \pi, n=0, \pm 1, \pm 2, \ldots, N$, where $n$ denotes the period of the orbit provided $N$ is the greatest integer satisfying $\cos \phi_{n}=n \pi(1-\varepsilon) /(1+\varepsilon) / \Gamma$, or equivalently $N<\Gamma(1+\varepsilon) /(1-\varepsilon) / \pi$. The stability of the fixed points $\left(\phi_{n}, v_{n}\right)$ of system (5) is identified by the determinant of the its Jacobian matrix:

$$
\left[\begin{array}{cc}
1 & 2 \\
-\Gamma(1+\varepsilon) \sin \left(\phi_{n}+2 v_{n}\right) & \varepsilon-2 \Gamma(1+\varepsilon) \sin \left(\phi_{n}+2 v_{n}\right)
\end{array}\right] .
$$

Since (6) is a real matrix, the eigenvalues can either be real or else exist as complex-conjugate pairs. A fixedpoint periodic orbit is linearly stable if and only if both eigenvalues of the stability matrix (6) lie inside the unit circle in the complex plane (see, for instance, $[1,8]$ ). The corresponding eigenvalues are found as $\lambda_{1,2}=[1+\varepsilon-\gamma$ $\left.\pm \sqrt{(1+\varepsilon-\gamma)^{2}-4 \varepsilon}\right] / 2$, where $\gamma=2 \Gamma(1+\varepsilon) \sin \left(\phi_{n}\right)$, with $\lambda_{1} \lambda_{2}=\varepsilon$. The parameter $0 \leqslant \varepsilon \leqslant 1$ is taken to be constant since we neglect its dependence upon the bounce velocity; for $\lambda_{1} \lambda_{2}=\varepsilon<1$, only sinks $\left(\left|\lambda_{1,2}\right|\right)<1$ and saddles $\left(\left|\lambda_{1}\right|<1\right.$ $<\left|\lambda_{2}\right|$ ) emerge from this system, while at $\varepsilon=1$ there appear centers and saddles. At a convenient value $\varepsilon=0.25$ to high- 


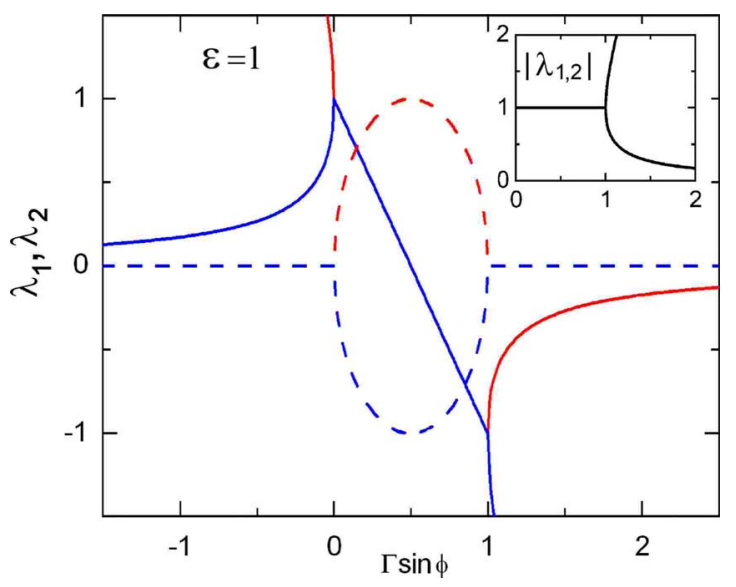

FIG. 3. (Color online) Behavior of the eigenvalues of system 5 at $\varepsilon=1$. In the inset, the eigenvalue magnitudes show that the system is linearly neutrally stable over the whole range $0 \leqslant \Gamma \sin \phi$ $\leqslant 1$.

light the peculiarities of the eigenvalue spectrum, the eigenvalues $\lambda_{1,2}$ are plotted against $\Gamma \sin \phi$ in Fig. 2, where the solid and dashed lines are for the real and imaginary parts of the eigenvalues, respectively. We see that the real branches are symmetrically connected by a straight line that crosses the horizontal axis at $(1 / 2,0)$ by joining the points $A=\left((1-\sqrt{\varepsilon})^{2} /[2(1+\varepsilon)], \sqrt{\varepsilon}\right)$ and $B=\left((1+\sqrt{\varepsilon})^{2} /[2(1+\varepsilon)]\right.$, $-\sqrt{\varepsilon})$, such that the projection of segment $A B$ on the horizontal axis gives a breadth of $2 \sqrt{\varepsilon} /(1+\varepsilon)$ bounded by the dashed closed curve onto which lie simple conjugate pairs of eigenvalues. Thus we see that for $\varepsilon=1$ (Fig. 3) the whole range $0 \leqslant \Gamma \sin \phi \leqslant 1$ corresponds to neutrally stable centers-i.e., $\left|\lambda_{1,2}\right|=1$. On the other hand, for the completely inelastic case $\varepsilon=0$, segment $A B$ collapses into the center point $(1 / 2,0)$ and the two curved branches turn into straight lines (Fig. 4). In the general case $(0<\varepsilon<1)$ as shown in Fig. 2, the left real branch intersects the vertical line $\Gamma \sin \phi=0$ at the points $C=(0,1)$ and $D=(0, \varepsilon)$, so that the locus of the magnitude of the eigenvalues crosses the unit circle in the complex domain at $\lambda_{1}=+1$, originating a saddle-node bifurcation. In a complimentary fashion the real right branch curves down and

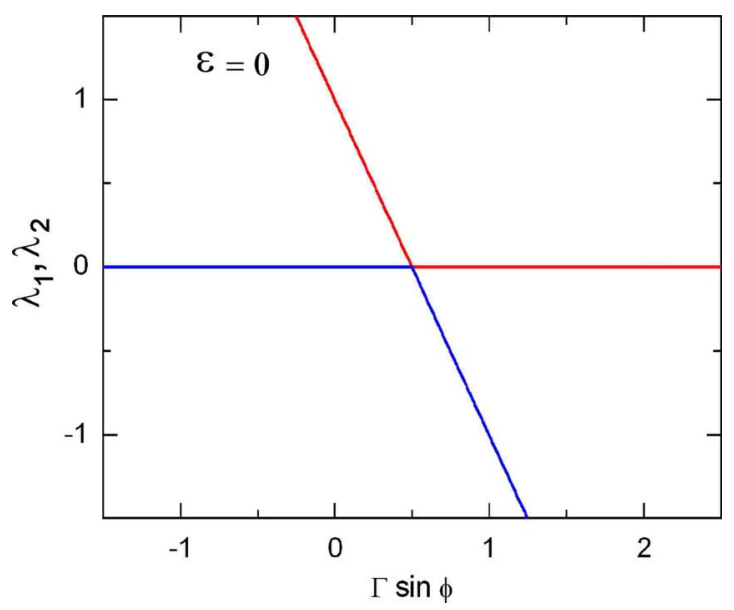

FIG. 4. (Color online) Eingenvalue behavior in the completely inelastic case $\varepsilon=0$.

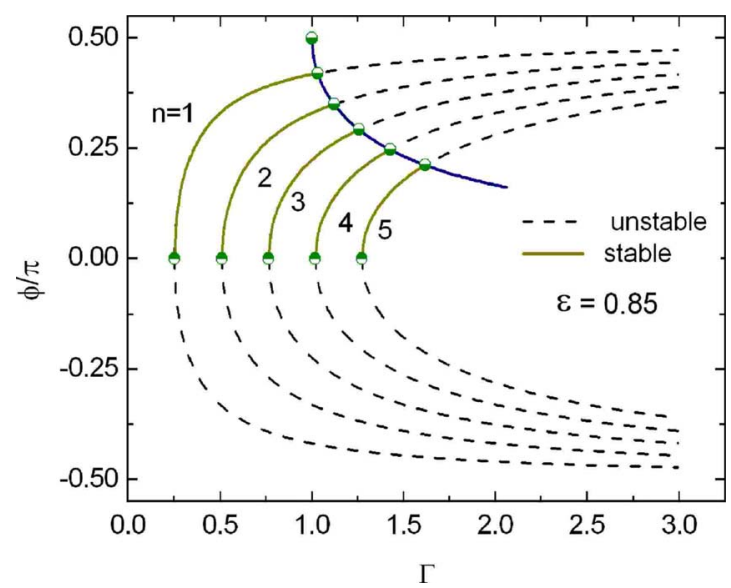

FIG. 5. (Color online) At $\varepsilon=0.85$, domains of stability for $n$-periodic orbits where $\phi$ denotes the phase at which the ball periodically bounces off the platform vibrating at driven amplitude $\Gamma$. The limiting upper curve is calculated from $\phi_{L}=\arcsin (1 / \Gamma)$, beyond which the mode evolves into its period-doubled version. Along the axis $\phi / \pi=0$, at which $\lambda_{1}=+1$, two periodic orbits are created: a saddle (unstable) and a node (stable).

intersects the vertical line $\Gamma \sin \phi=1$ at $E=(1,-\varepsilon)$ and at the period-doubling bifurcation point $F=(1,-1)$. As a result of bifurcation, the stable orbit loses its stability and spawns a period- 2 orbit since this time the eigenvalue crosses the unit circle at $\lambda_{2}=-1$. Therefore, on the left half-plane $\Gamma \sin \phi$ $<0$ lie saddle points of the first kind with positive eingenvalues $\left(0<\lambda_{1}<1<\lambda_{2}\right)$, which render the periodic orbits unstable. Saddles of the second kind with negative eigenvalues $\left(\lambda_{1}<-1<\lambda_{2}<0\right)$ are found on the right half-plane bounded by the treshold line $\Gamma \sin \phi=1$, and sinks (or centers) with $\left|\lambda_{1,2}\right|<1$ are confined in the range $0<\Gamma \sin \phi<1$ (Fig. 2).

To explicitly determine the lower and upper bounds in terms of the coefficient of restitution $\varepsilon$, we use Eq. (5b)i.e., $\Gamma \cos \phi=n \pi(\varepsilon-1) /(\varepsilon+1)$ - which gives the threshold and limiting values of $\Gamma$ whose associated eigenvalues are sinks $\left(\left|\lambda_{1,2}\right|<1\right)$ : namely,

$$
n \pi \frac{1-\varepsilon}{1+\varepsilon}<\Gamma<\sqrt{1+n^{2} \pi^{2}\left(\frac{1-\varepsilon}{1+\varepsilon}\right)^{2}},
$$

where the left inequality is the onset condition for the $n$th subharmonic to be generated, with the right inequality ensuring its stability.

Correspondingly, the fixed points are saddles of the second kind $\left(\lambda_{1}<-1<\lambda_{2}<0\right)$ if

$$
\Gamma>\sqrt{1+n^{2} \pi^{2}\left(\frac{1-\varepsilon}{1+\varepsilon}\right)^{2}} .
$$

Calculated for $\varepsilon=0.85$, the branches of stability of periodic orbits with subharmonic number $n$ are shown in Fig. 5, where the limiting curve is given by $\phi_{L}=\sin ^{-1}(1 / \Gamma)$. Determined from Eq. (7), the two sets of open circles denote bifurcation values, the first of which (along the line $\phi=0$ ) appears in a saddle-node bifurcation, while the second indicates a change of stability followed by a period doubling bifurcation $[11,17]$. 


\section{DYNAMICAL EQUATIONS AND MODE STABILITY}

In this section we derive the initial starting condition for the ball to execute a periodic motion upon collision with the vibrating platform. Then we discuss the various characters of the bouncing ball trajectories determined numerically from the full system (5). The numerical solutions are obtained by using an event-driven procedure [18] that consists in monitoring a sequence of events for which the force and trajectory equations (5) are solved without resorting to any approximation.

Consider a ball dropped at zero initial velocity from the actual height $H_{0}$, at the initial phase $\phi_{0}$. In nondimensionalized coordinates $h_{0}=H_{0} \omega^{2} / g$, the free flight is described by $h=h_{0}-\left(\phi-\phi_{0}\right)^{2} / 2$, such that at the collision phase $\phi$, the height is $h=\Gamma \sin \phi$, and since for a periodic motion of subharmonic index $n$ the initial phase $\phi_{0}$ is symmetrically spaced from the phases for the $(n-1)$ th and $n$th impacts, the relation

$$
\phi=\phi_{0}+n \pi
$$

holds, and therefore

$$
h_{0}=\frac{1}{2}(n \pi)^{2}+\Gamma \sin \phi .
$$

The exact phase at which impact occurs is such that the upward movement of the platform compensates for the energy loss from the inelastic collisions so that the ball lands and departs from the platform at the same speed $v=n \pi$ (relative to the laboratory frame), which is consistent with the final velocity the ball reaches after the time interval $\phi-\phi_{0}$ $=n \pi$ given in Eq. (9). Recalling that the coefficient of restitution $\varepsilon$ relates to $\Gamma$ and $\phi$ through

$$
\Gamma \cos \phi=n \pi \frac{1-\varepsilon}{1+\varepsilon} .
$$

Then, for a given $\varepsilon$ and period index $n$, the initial height $h_{0}$ and phase $\phi_{0}$ are calculated from Eqs. (9)-(11) provided the constraint $0<\Gamma \sin \phi<1$ is fulfilled to ensure stability of the

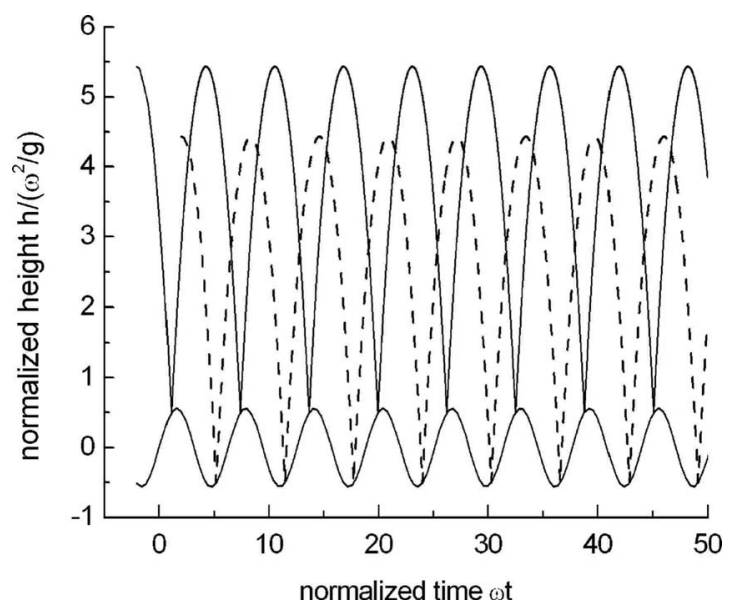

FIG. 6. For $\varepsilon=0.85$, period- 1 modes driven at $\Gamma=0.5611$ with collision phases $\phi=0.3501 \pi\left[h_{0}=\left(\pi^{2}+1\right) / 2\right.$, solid line $]$ and $\phi=-0.3501 \pi\left[h_{0}=\left(\pi^{2}-1\right) / 2\right.$, dashed line $]$.

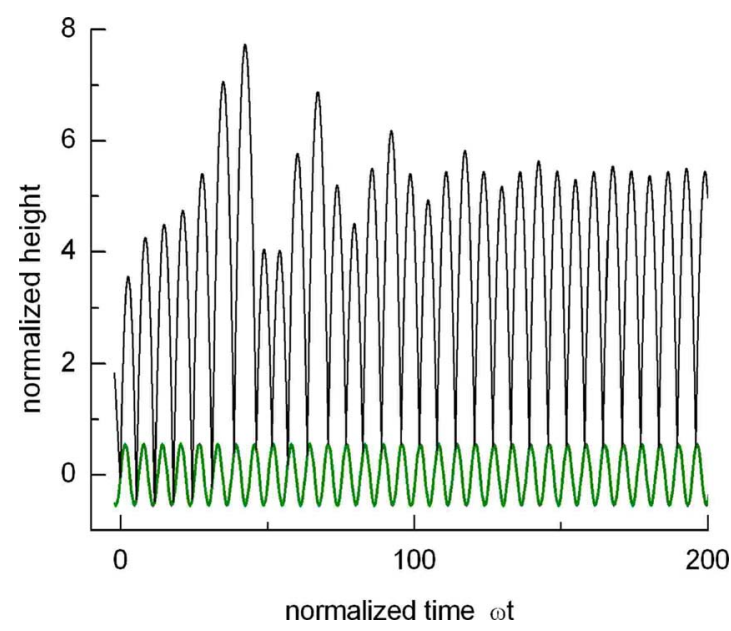

FIG. 7. (Color online) Dropped from the height $h_{0}=1.823$ at $\phi=-0.6499 \pi$, the ball enters the period- 1 mode after an initial transient. The lower wavy curve depicts the sinusoidal vibration of the driven platform.

periodic orbits. Assuring the existence of periodic orbits, Eq. (11) has two solutions, one of which is unstable as will be discussed next. To this end, we set $\Gamma \sin \phi=1 / 2$ at $\varepsilon=0.85$ and $n=1$, thus resulting in a complex pair of eigenvalues $\lambda_{1,2}= \pm i \sqrt{\varepsilon}$. Using Eq. (11) gives $\Gamma=0.5611$ and $\phi$ $= \pm 0.3501 \pi$, which combined with Eqs. (9) and (10) yields two solutions with the corresponding starting conditions $\left[\phi_{0}=-0.6449 \pi, \quad h_{0}=\left(\pi^{2}+1\right) / 2\right.$ and $\phi_{0}=0.6449 \pi, \quad h_{0}$ $\left.=\left(\pi^{2}-1\right) / 2\right]$. Of course, dropped at such consistent starting parameters the ball immediately enters the period- 1 modei.e., without overshoot or transient as shown in Fig. 6. Similar behavior is exhibited by the second (and unstable) solution indicated by the dotted line. Concerning the first solution, it shows a robust stability with respect to initial heights ranging in the interval as wide as [1.823, 11.510].

To illustrate this point, by dropping from the height $h_{0}$ $=1.823$, with the remaining parameters kept unchanged, the ball settles down to equilibrium through a sequence of jumps (Fig. 7) to reach the final position at the converging point $(0.3500 \pi, 1)$ in phase space $(\phi / \pi, v / \pi)$ shown in Fig. 8. We

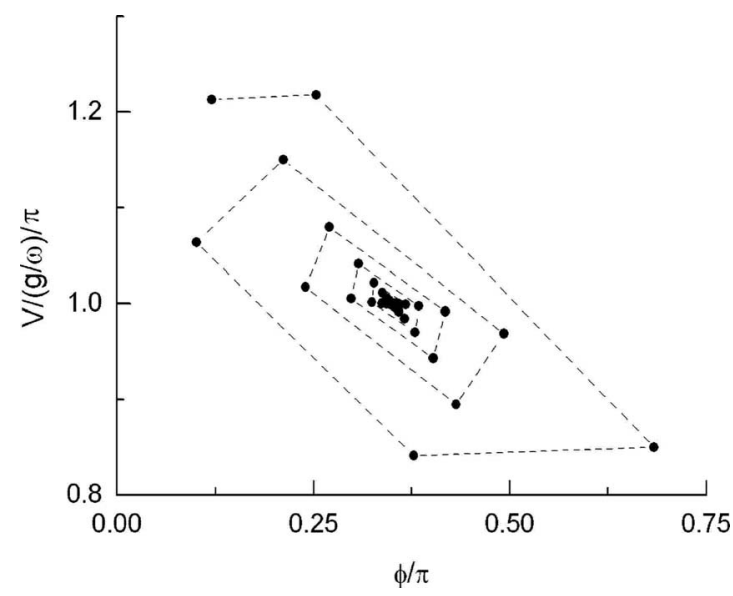

FIG. 8. Phase-space plot of the period-1 mode with behavior shown in Fig. 7. 


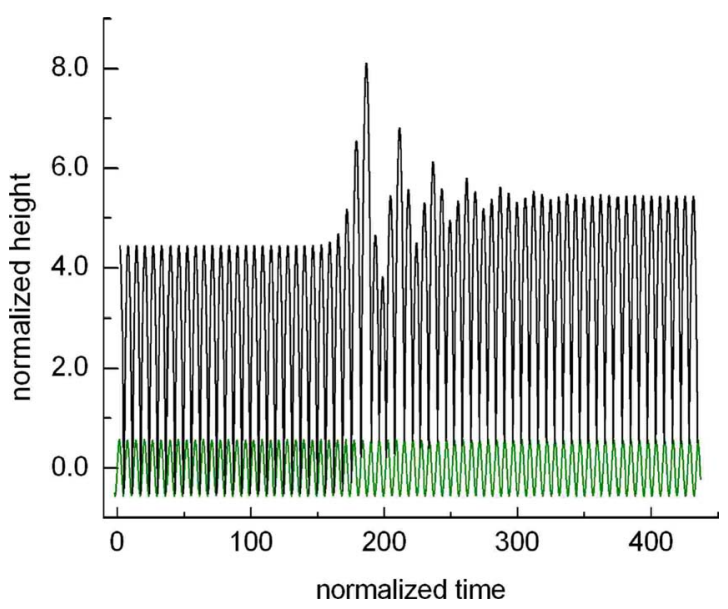

FIG. 9. (Color online) Dropped from the height $h_{0}=4.4348$ at $\phi_{0}=0.6499 \pi$, the ball switches from the unstable mode to its stable counterpart after suffering a correction on its collision phase.

see in the transient behavior that the phase suffers corrections after a succession of collisions on the ascending phase to be locked in the period-1 mode at the periodic collision phase $\phi / \pi=0.3501$; calculated using Eq. (11), such a value agrees within a $0.01 \%$ accuracy with that from the eventdriven procedure.

For the second solution at the initial time, or equivalently at the initial phase $\phi_{0}=0.65001 \pi$ slightly lagging relative to the correct phase $\left(\phi_{0}=0.6499 \pi\right)$, Fig. 9 explains the unstable character of the second solution, for which a slightly delayed collision occurs when the table in its upward movement is rising faster, which makes the ball rise to a larger height so as to arrive for the next collision still further delayed. As the ball starts jumping higher and higher, it eventually lands when the table is moving away in a descending phase, and thereafter the collision phase suffers a correction, leading the ball to be locked in its counterpart stable mode.

At $\phi_{0}=0.65001 \pi$, now dropping the ball from a slightly lower initial height $h_{0}=1.822$ (just on the left limit of the stability range for $h_{0}$ ), in this case the ball is unable to sus-

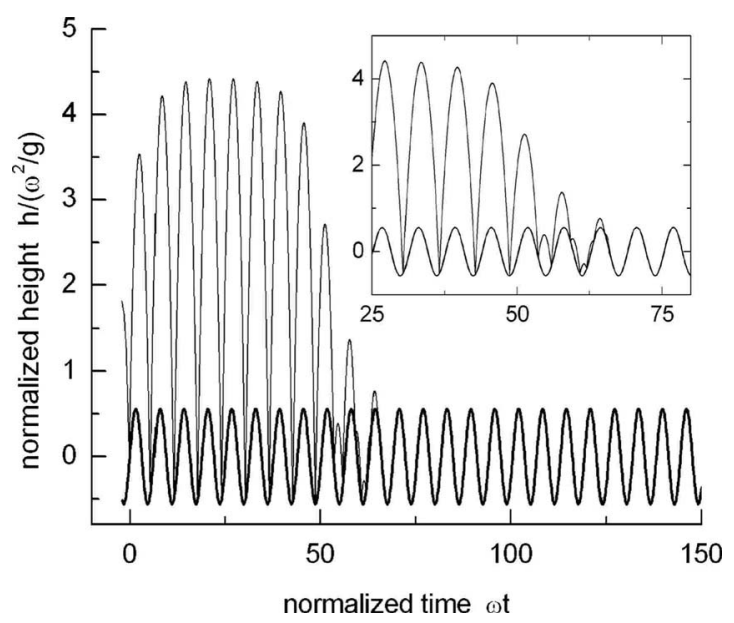

FIG. 10. Differently from Fig. 7, the ball dropped from $h_{0}$ $=1.822$ is unable to enter the period -1 mode. Transient motion is zoomed in on in the inset.

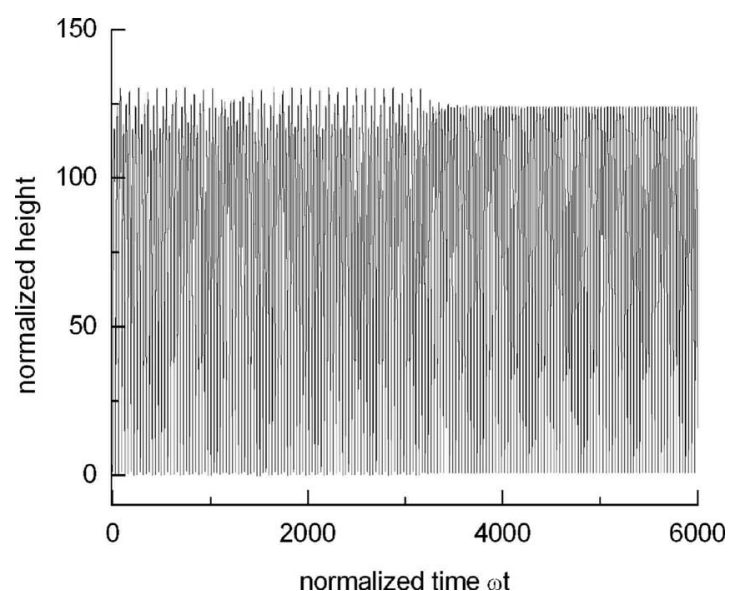

FIG. 11. Dropped from $h_{0}=132.0$, at $\varepsilon=0.85$ and $\Gamma \sin \phi=0.8$ with $\Gamma=1.504$, the ball reaches the period-5 mode.

tain its motion and then comes to a permanent contact with the platform by executing a convergent sequence of decaying jumps. As shown in Fig. 10, after the tenth collision the impact position commutes to a descending phase when the base, moving downward, has a negative velocity. The ball loses energy, and in the next collision the ball, upon rising to a lower height, arrives further delayed; the lost synchronism cannot be restored, and the ball rests immobile on the platform.

To drive a higher-order $n$ th-subharmonic periodic-1 mode from required specifications-for instance, $n=5$ at $\varepsilon=0.85$ and $\Gamma \sin \phi=0.8$ - we use Eqs. (9)-(11) to consistently obtain the starting parameters $\phi_{0}=-4.821 \pi, h_{0}=(\Gamma \sin \phi$ $\left.+(5 \pi)^{2} / 2\right)=124.17$, and the drive amplitude $\Gamma=1.504$ by noting that the collision phase $\phi=0.1785 \pi$ relates to the initial phase by $\phi=\phi_{0}+5 \pi$. Instead of dropping the ball from the calculated height $\left(h_{0}=124.17\right)$, at which the ball would enter the $n=5$, period- 1 motion without transient, let us drop the ball from a larger height $h_{0}=132.0$. Preceded by a per-

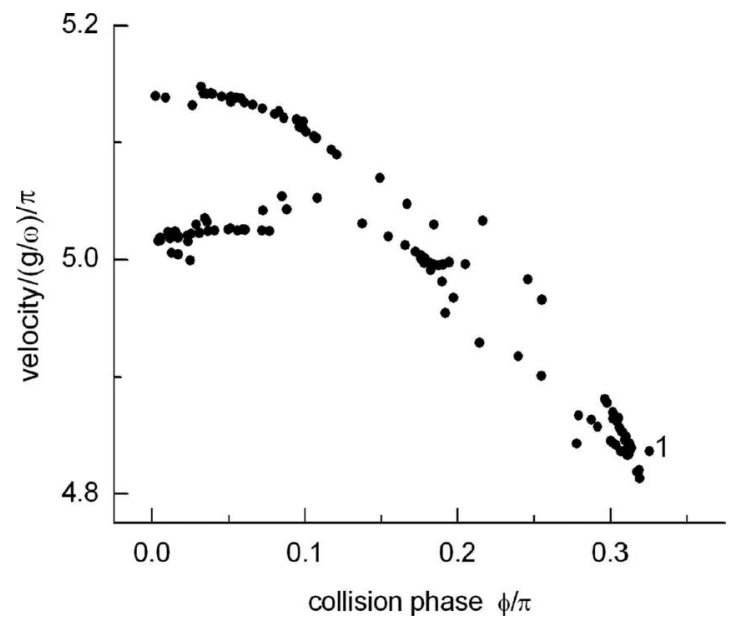

FIG. 12. Phase-space plot of the ball motion shown in Fig. 11. The system evolves to the converging point $(\phi / \pi, v / \pi)$ $=(0.1775,5)$ identifying a $n=5$ periodic mode; the three clusters of points are remnants from the period-tripling transient time series displayed in Fig. 11. 


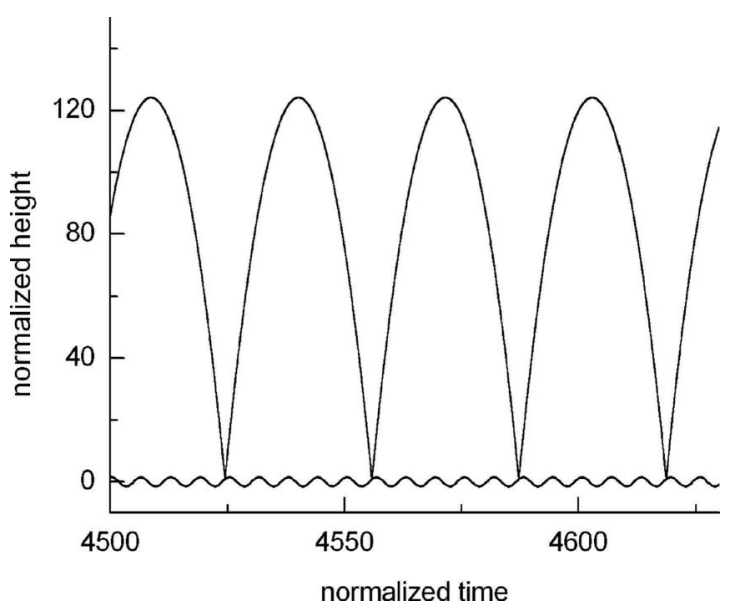

FIG. 13. Periodic mode with subharmonic index $n=5$ : both the landing and departure velocities are $5 \pi$, and two consecutive collision phases are spaced by $5 \pi$.

sistent sequence of somehow period-tripling oscillations, as shown in Fig. 11, the ball ultimately reaches its steady state of motion past the time span of nearly $3500 / 2 \pi$ oscillation periods through exponentially damped oscillations, as in this case the resulting eigenvalues $\lambda_{1,2}=0.5500 \pm i 0.7362$ typify a stable focus. After the transient is finished, the ball reaches the collision velocity $v=5 \pi$ at the phase $\phi=0.1785 \pi$, as portrayed in the phase-space plot in Fig. 12. Detailed in Fig. 13, the steady-state oscillations consist of equal jumps separated by a periodic time span of $5 \pi$; each jump is 25 times as high as the single jump in the $n=1$ periodic mode discussed in Fig. 6. Here we note that for a $n$ th-subharmonic periodic mode the maximum height relative to the impact point is just $(n \pi)^{2} / 2$, irrespective of the drive amplitude $\Gamma$. In spite of increasing $\Gamma$ the relative height remains constant; otherwise, the ball would start jumping higher, thus leading to longer flights which would not be synchronized with the oscillation period of the platform. In preserving its relative height to the collision point, the wavetrain of parabolic jumps shifts as a whole by searching for a new footpoint so as to keep both the landing and departure velocities matched at the synchronous value $v=n \pi$. This statement is expressed geometrically

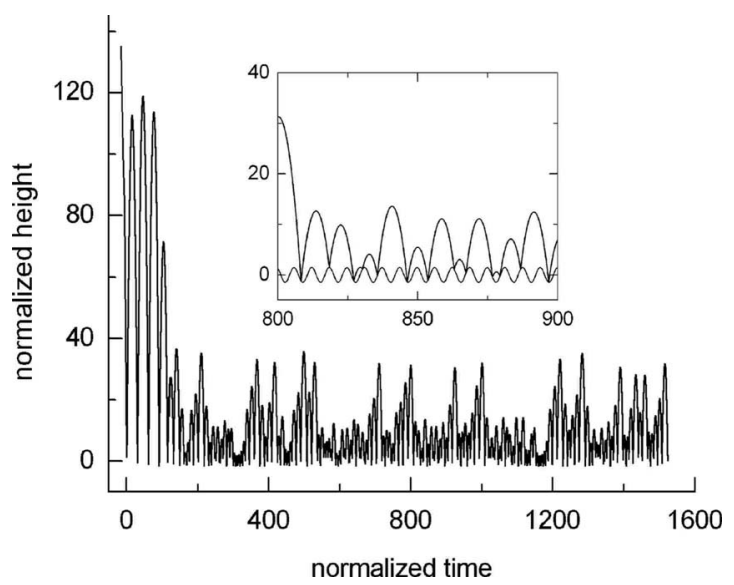

FIG. 14. At $\varepsilon=0.85$ and $\Gamma \sin \phi=0.8$ with $\Gamma=1.504$, but dropped from $h_{0}=135.0$ the ball enters a chaotic motion.

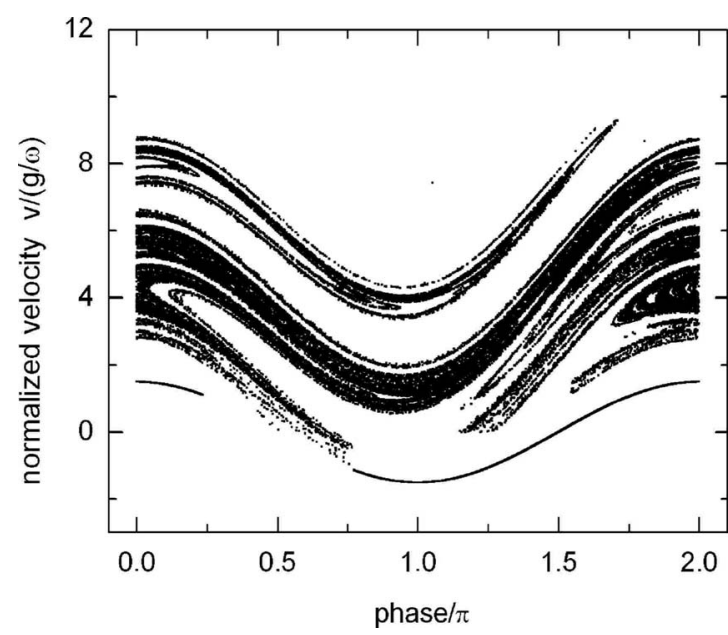

FIG. 15. Phase-space plot of the chaotic motion in Fig. 14.

by the stable branches shown in Fig. 5, where each branch, at a given mode index $n$ and an assigned $\varepsilon$, follows the constraint $\Gamma \cos \phi=n \pi(1-\varepsilon) /(1+\varepsilon)=$ const.

Without changing the previous control parameters $\Gamma$ and $\varepsilon$, now dropping the ball from a bit larger height-namely, $h_{0}=135.0$ - chaotic oscillations are generated (Fig. 14). Chaotic trajectories do not fill the phase space in a random manner. As seen in the map (Fig. 15) encompassing many unstable orbits which remain in the system, the trajectories fall onto a complex but well-defined and bounded object (chaotic attractor) which is cut at the bottom by a cosine-shaped boundary rendered by the velocity time profile $\left(\Gamma \cos \omega t_{i}\right.$ with $\omega t_{i}=\phi_{i}$ ) of the table oscillation. That the orbits remain bounded can be seen from Eq. (5)-i.e., $\left|v_{i+1}\right| \leqslant \mid \varepsilon v_{i}$ $+\Gamma \cos \phi_{i}|\leqslant \varepsilon| v_{i} \mid+\Gamma$; provided that $\Gamma<\left|v_{i}\right|(1-\varepsilon)$, this gives $\left|v_{i+1}\right| \leqslant\left|v_{i}\right|$, implying that all orbits stay confined in a strip $v= \pm \Gamma /(1-\varepsilon)$. At $\Gamma=1.504$ and $\varepsilon=0.85$ one finds $|v|$ $=10.027$, which is in good agreement with the velocity range [-1.504, 9.291] portrayed in Fig. 15 .

Discussing now the perfectly inelastic case $\varepsilon=0$, and according to Eq. (5b), the relative take-off velocity $v_{i+1}-v_{\text {base }}$

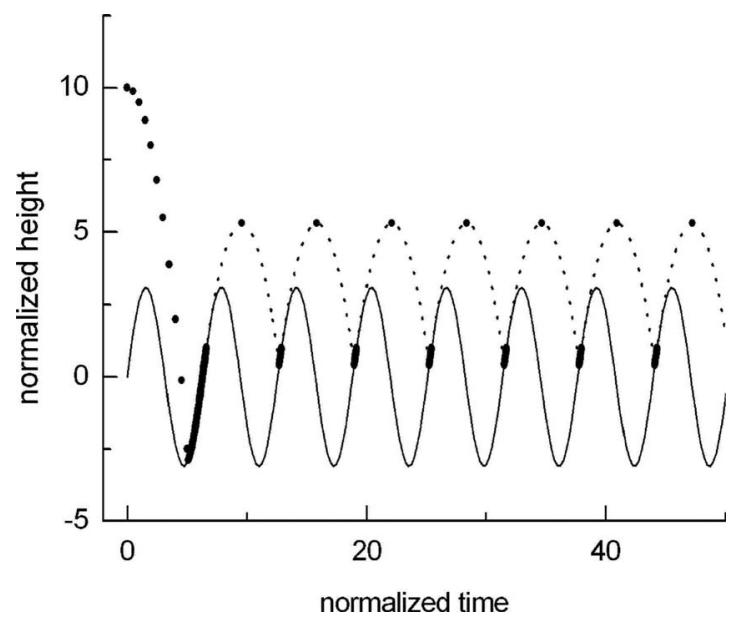

FIG. 16. Motion at $\varepsilon=0, \phi_{0}=0, h_{0}=10.00$, and $\Gamma=3.1$. The ball (solid circles) waits on the platform until $\Gamma \sin \phi=1$, the instant at which the ball is relaunched with a positive velocity and the process repeats itself periodically. 


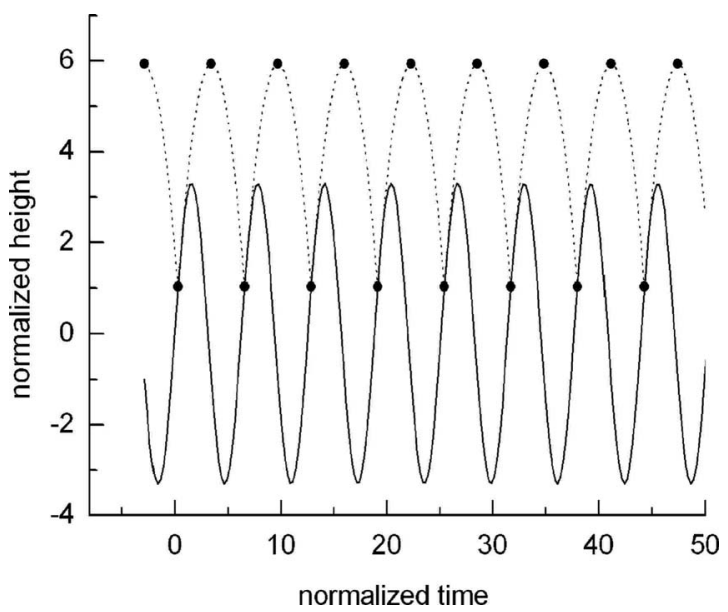

FIG. 17. Period-1 mode at the completely inelastic case $\varepsilon=0$. The starting parameters $\phi_{0}=-0.9019 \pi, h_{0}=10.00$, and $\Gamma=3.296$ are consistently calculated for the ball to depart without sticking on the platform.

of the ball vanishes identically. After impact, therefore, the ball acquires as its own velocity that of the platform. Thus losing all memory of its earlier velocity the ball sits on the platform and waits there until the platform's downward acceleration equals the gravity; having reached this condition, the ball is ejected from the platform on its ascending phase. This situation is shown in Fig. 16, on using $\Gamma=3.1$ and $\phi_{0}=0$.

Dropped from $h_{0}=10.00$, the ball hits the platform and remains sitting there for nearly one-quarter of a cycle until at $t_{2}=6.618$ the ball flings off the platform. During the waiting period, the ball is unable to depart inasmuch the platform is rising with a positive velocity because the base acceleration is still less than that of gravity, with the dimensionless value of -1 . Quantitatively, the acceleration $-\Gamma \sin \left(\phi+\phi_{0}\right)$ of the base equals -1 at the departure phase $\phi_{2}=0.1045 \pi$, calculated by $\Gamma \sin \left(\phi_{2}-2 \pi\right)=1$, and hence the take-off velocity is $\Gamma \cos \phi_{2}=2.9343$, a value $0.3 \%$ above that calculated by the event-driven procedure. In the present example and calculated as $\left(v^{2} / 2\right)-\Gamma \sin \phi_{2}$, the maximum height relative to the

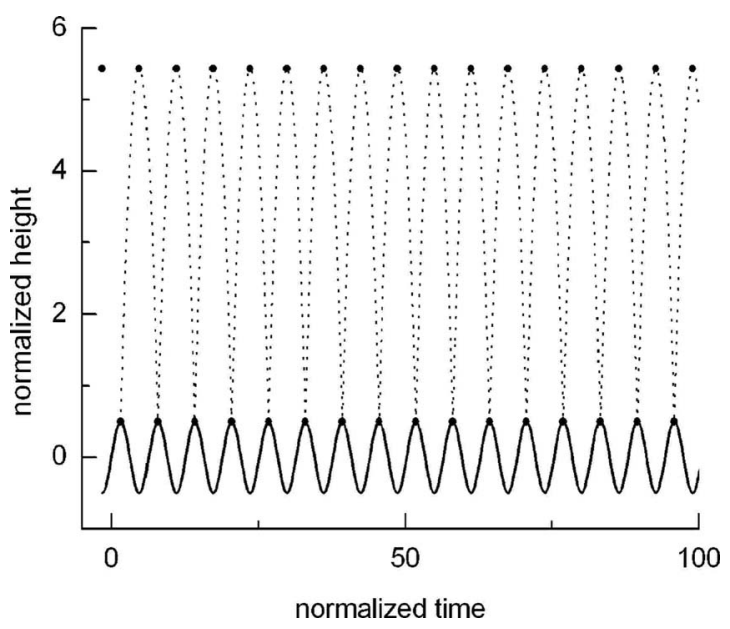

FIG. 18. Period-1 mode at $\varepsilon=1(\Gamma=0.5, \phi=\pi / 2)$.

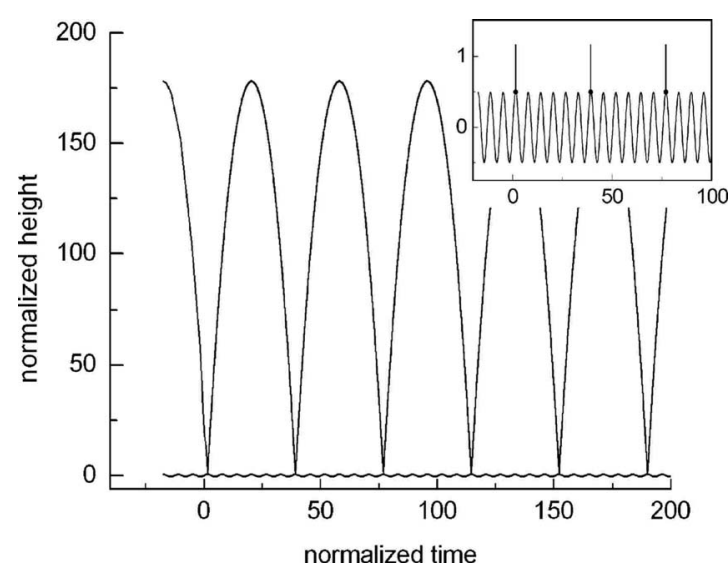

FIG. 19. Sixth subharmonic mode at $\varepsilon=1$. The inset shows that two consecutive collisions are spaced by six periods of the base oscillation.

impact point is 4.304, which is shorter than the height of $\pi^{2} / 2=4.934$ for a pure period- 1 mode, for which the preand post-impact velocities are the same. For exciting such a pure mode, we make $\Gamma \sin \phi=1$ and use $\Gamma \cos \phi=\pi$ [obtained from Eq. (5b)] and $\Gamma \cos \phi=n \pi(1-\varepsilon) /(1+\varepsilon)$, at $\varepsilon$ $=0$, to obtain the drive amplitude $\Gamma=3.296$ and $\phi=0.098 \pi$. Dropped from the same height $h_{0}=10.00$ as before (Fig. 16), but at $\phi_{0}=0.9019 \pi(=\pi-\phi)$ and $\Gamma=3.296$, the resulting trajectory undergoes single 1-periodic jumps without the ball staying in contact with the base, as shown in Fig. 17.

In concluding this section, we discuss the elastic case $\varepsilon=1$, by considering first the equation $v_{2}=\varepsilon v_{1}+(1$ $+\varepsilon) \Gamma \cos \phi$ that relates the pre- and post-collisional velocities of the ball upon impact against the moving platform, where $v_{1}>0$ denotes the incoming velocity. Then, at $\varepsilon=1$ and for head-on collisions, in which the base moves upwards, the post-collisional reduces to $v_{2}=v_{1}+2 \Gamma \cos \phi$, while for overtaking collisions (with the base moving downwards) the post-velocity turns into $v_{2}=v_{1}-2 \Gamma \cos \phi$, where $\phi$ is the collision phase. If the post-collision velocity is negative (particle still moving down), then a second impact will occur, provided that $V<v_{1}<2 V$, where $V=|2 \Gamma \cos \phi|$. But

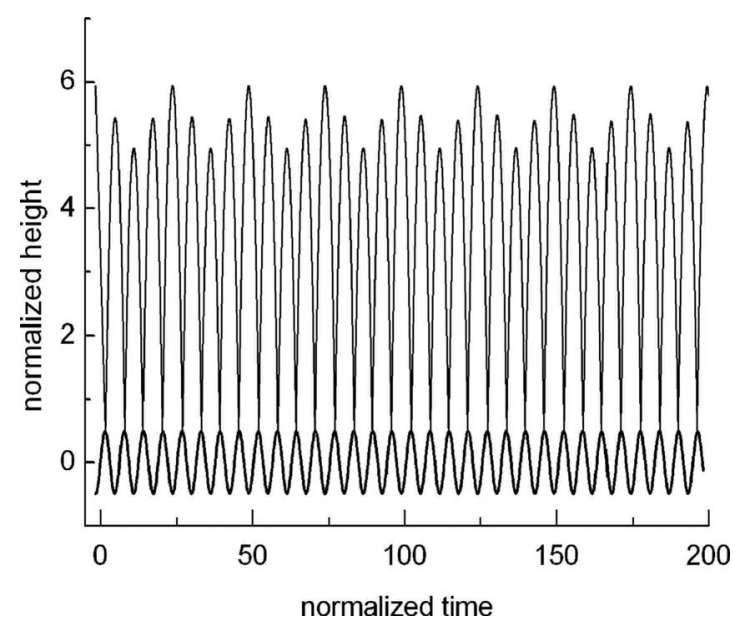

FIG. 20. Amplitude modulated version of the period-1 mode at $\varepsilon=1$. 


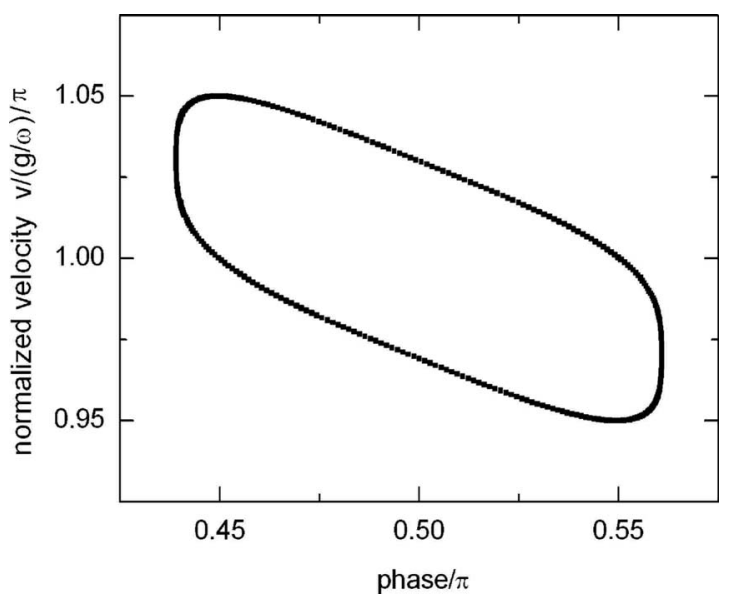

FIG. 21. Phase-space plot of the motion in Fig. 20.

for both types of collisions, when $\phi=n \pi / 2, n$ integer, the ball bounces back after collision with a departure velocity, which is simply the reverse of the velocity before the bounce, neither gaining nor losing energy on the collision. For the period- 1 mode, at $\phi_{0}=-\pi / 2, \Gamma=0.5$, and $h_{0}=\pi^{2} / 2$ $+\Gamma$, this situation is shown in Fig. 18. At such starting conditions, the time length between collisions is matched with the period of the platform motion, with the ball performing a sequence of perfectly equal jumps and seeing the base as it were static. Accordingly, by dropping the ball from $h_{0}$ $=(6 \pi)^{2} / 2+\Gamma$ at $\phi_{0}=-11 \pi / 2$, the ball will execute a $n=6$ subharmonic motion (Fig. 19), in which two consecutive collisions are spaced by six periods of the base oscillation.

On the other hand, when the extra term $Z$ in $h_{0}=\pi^{2} / 2$ $+Z$ differs from the oscillation amplitude $\Gamma$ as required for driving periodic modes, the resulting trajectories become amplitude modulated as pictured in Fig. 20, where $\Gamma=0.5$ and $Z=10$. In its phase-space plot (Fig. 21) the motion appears as an ellipticlike curve enclosing the fixed point $(\phi, \pi)=(\pi / 2, \pi)$.

Releasing now the ball from $h_{0}=0.5$ at $\Gamma=0.5$ and $\phi_{0}$ $-\pi / 2$, we see in Fig. 22 that the jumps starts increasing

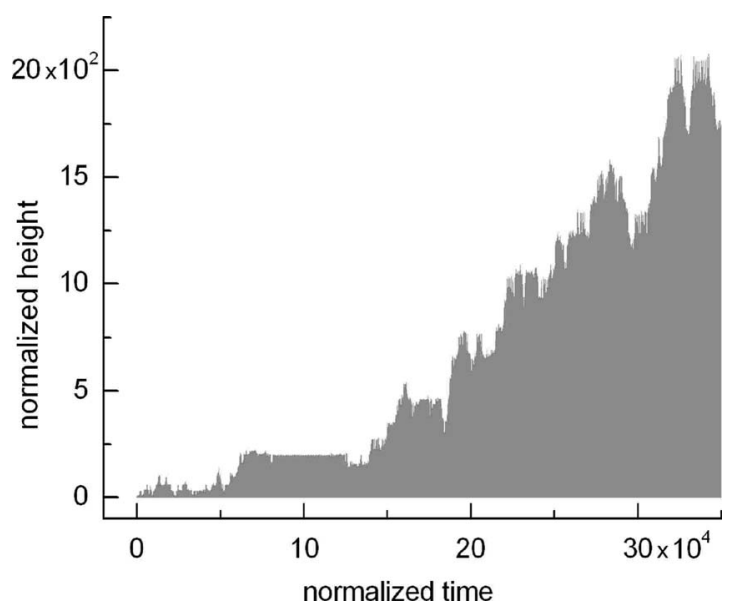

FIG. 22. At $\varepsilon=1$ with the start conditions $h_{0}=0.5, \phi_{0}=-\pi / 2$, and $\Gamma=0.5$, the amplitude of the period- 1 mode shows a boundless increase.

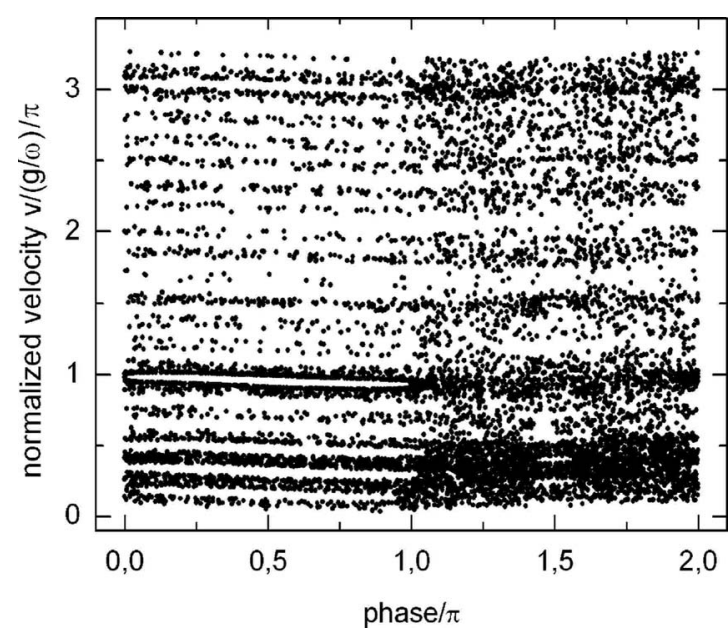

FIG. 23. Phase-space plot of the motion in Fig. 22.

without limit, an example that exhibits Fermi acceleration - a process in which the particle gains energy by collision against a moving scatterer. Gain or loss of energy occurs on head-on (base moves towards the incoming particle) and overtaking collisions (base moves away from the particle), but the net result will be an average gain by the reason that increasing velocities make head-on collisions more frequent. In fact, averaging from $v_{2}=v_{1}+2 \Gamma \cos \phi$ the square velocity $v_{2}$ over an oscillation period leads to $\left\langle v_{2}^{2}\right\rangle$ $=\left\langle v_{1}^{2}\right\rangle+2 \Gamma^{2}$, which describes a constant net energy gain per collision, thus meaning that the particle's energy (or height) tends to increases linearly with time. This is shown in Fig. 22 , where the increase of the particle energy roughly follows a linear growth, thus characterizing a random walking particle, for which the average velocity scales with the collision number as $\langle v\rangle \approx \sqrt{N}$ [5]. In the phase-space plot (Fig. 23) of this motion, there appear stochastic layers, which, separated from each other, seem to be limited in their width.

To examine some stability issues at $\Gamma=1.1$ and $\phi=\pi / 2$, we set the initial conditions as $h_{0}=(6 \pi)^{2} / 2+0.5$ and $\phi_{0}$ $=-11 \pi / 2$ and obtain the motion pictured in Fig. 24, where we see that the amplitude still remains modulated up to $\approx 10^{4}$

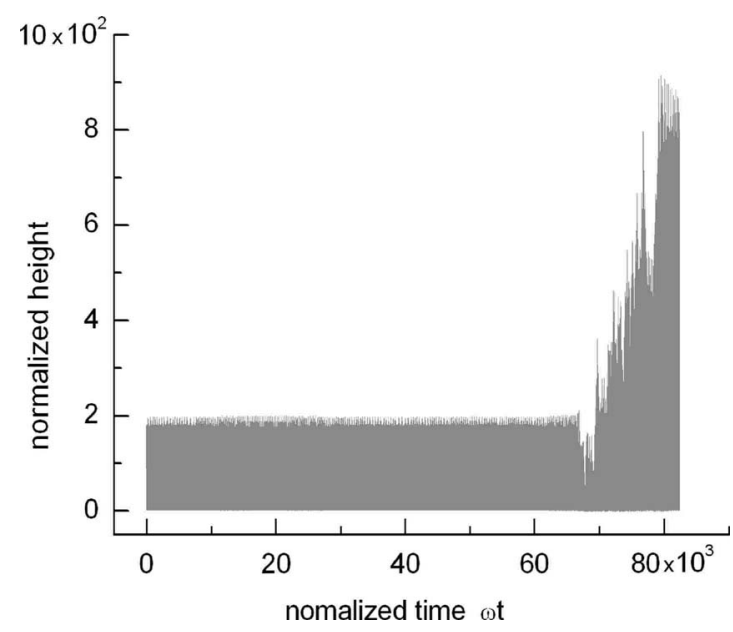

FIG. 24. Motion at $\varepsilon=1$ and $\Gamma=1.1$ with starting conditions $h_{0}=(6 \pi)^{2}+0.5$ and $\phi_{0}=-11 \pi / 2$. 


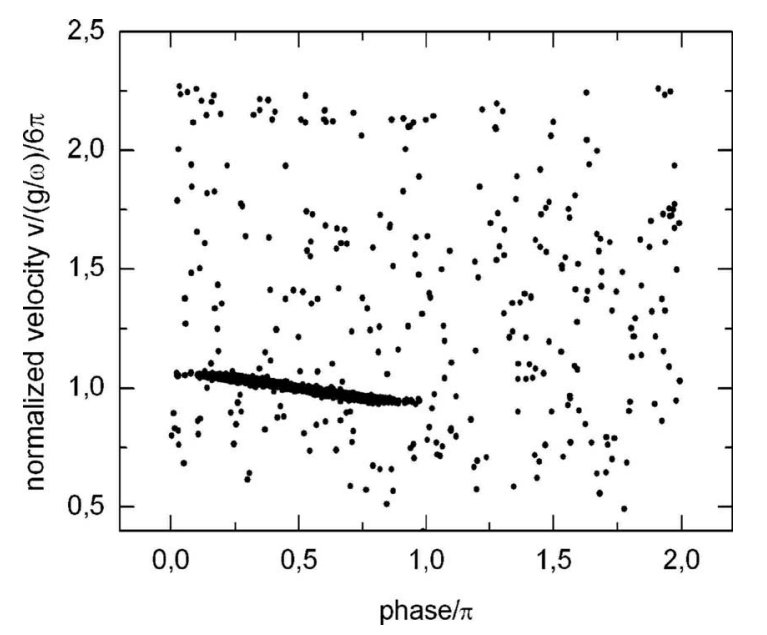

FIG. 25. Phase-space plot of the motion in Fig. 24.

periods of the base oscillation. After that span of time the mode destabilizes and the ball goes jumping higher and higher. Characteristically asymmetric, the associated phasespace plot (Fig. 25) shows an elliptic-shaped structure (constrained in the phase interval $0<\phi / \pi<1$ ) surrounded by scattered points. A zoomed-in view (Fig. 26) of such an oblong structure (up to the running time of $63.3 \times 10^{3}$ ) reveals a pinched ellipsis centrally filled with a cloud of points. Moreover, the cloud is centered at $(\phi, v)=(\pi / 2,6 \pi)$, which, appearing as an $n$ attracting center, just corresponds to the stable fixed point of the $n=6$ period -1 motion. This suggests that the scattered points are associated with the growingamplitude motion that develops after the period- 6 mode becomes unstable. From this motion $(\Gamma=1.1, \phi=\pi / 2, \varepsilon=1)$ emerge the eigenvalues $\lambda_{1}=-0.5367$ and $\lambda_{2}=-1.863$ (often categorized as a hyperbolic fixed point since both of them lie off the imaginary axis), which correspond to an attracting node, thereby explaining the robust stability of the period- 6 mode, which remained stable for $10^{4}$ oscillation periods. In the previous cases $(\Gamma=0.5, \phi=\pi / 2)$, by contrast, their associated eigenvalues all are a center $\left(\lambda_{1,2}= \pm i\right)$, thus characterizing fragile trajectories, which are affected by small perturbations, which make the motion depart from the equilibrium.

\section{CONCLUSIONS}

Through numerical examples from computer simulations guided by analytical considerations, this paper has presented a quantitative description of the bouncing ball problem. The dynamics is modeled on the basis of a discrete map of difference equations for the trajectory (describing the ball free fall under gravitational acceleration), velocity, and phase of the ball's motion on assuming a constant restituition coefficient and the collisions to be instantaneous.

Once iterated numerically, the equations fully reveal a rich variety of nonlinear behaviors, including nonperiodic motions as well as chaotic and stochastic phenomema. In the context of the high-bounce approximation [the landing velocity at the $(i+1)$ th collision is the reverse of the of the take-off velocity of the prior collision-i.e., $\left.U_{i+1}=-V_{i}\right]$ and

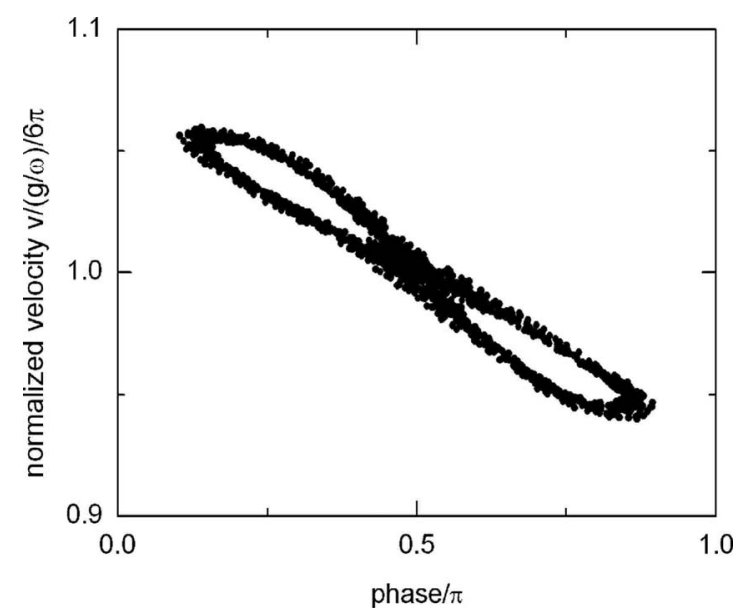

FIG. 26. A zoomed-in view of the phase-space plot in Fig. 25.

in the case of periodic motion (in which the ball makes identical jumps upon collision with the table at the same phase of the ball's motion), the system simplifies to a pair of equations. Holding exactly for periodic motions, the reduced system is then linearized about the fixed point to give a characteristic equation from which the stability and bifurcation conditions are determined and expressed as $0<\Gamma \sin \phi<1$, showing that asymptotic stability is ensured when the collision phase lies in the range $0<\phi \leqslant \pi / 2$. Outside the stability range, two types of unstable motion exist for period-1 orbits-saddle of the first kind for $\pi / 2<\phi<0$ and saddle of the second kind for $\Gamma \sin \phi>1-$ while the boundaries between stable and unstable solutions define the onset of bifurcations: period doubling at $\Gamma \sin \phi=1$ and saddle-node bifurcation at $\Gamma \sin \phi=0$.

Following such analytical considerations, numerical examples have demonstrated that the bouncing ball behavior is strongly dependent on the control parameters ( $\Gamma$ and $\varepsilon$ ) and also on the initial conditions. At correct initial values of the height $\left(h_{0}=n \pi^{2} / 2+\Gamma \sin \phi\right)$ and phase $\left(\phi_{0}=\phi-\pi\right)$, the ball is instantaneously locked in the periodic modes. For instance, setting $\Gamma \sin \phi=1 / 2, n=1$, and $\varepsilon=0.85$, the correct initial conditions for such a period-1 mode are calculated as $\phi_{0}=0.6499 \pi$ and $h_{0}=5.4348$. But dropping the ball from $h_{0}=1.822$ (while keeping the remaining parameters constant) leads to chattering, by which the ball gets locked onto the table through a sequence of decaying jumps, while at $h_{0}$ $=1.823$ the ball motion still evolves to period -1 motion. At $\Gamma \sin \phi=0.8, \varepsilon=0.85, h_{0}=132.0$ the ball reaches $n=5$ subharmonic motion after executing a long-lasting sequence of period-tripling oscillations. Dropped from $h_{0}=135.0$, by contrast, there appear irregular periodic orbits that converge toward a strange attractor bounded by the velocity strip $v= \pm \Gamma(1-\varepsilon)$.

\section{ACKNOWLEDGMENT}

This work has been supported by the National Council for Scientific and Technological Development (CNPq), Brazil. 
[1] A. B. Pippard, The Physics of Vibration, Omnibus ed. (Cambridge University Press, New York, 2006), Chap 9.

[2] G. M. Zaslavski and B. V. Chirikov, Sov. Phys. Usp. 14, 549 (1972).

[3] J. M. Pastor, D. Maza, I. Zuriguel, A. Garciamartín, and J.-F. Boudet, Physica D 232, 128 (2007).

[4] E. Fermi, Phys. Rev. 75, 1169 (1949).

[5] E. D. Leonel and A. L. P. Livorati, Physica A 387, 1155 (2008).

[6] J. M. T. Thompson and H. B. Stewart, Nonlinear Dynamics and Chaos (Wiley, New York, 1991), Chap. 14.

[7] N. A. Burnham, A. J. Kulik, and G. Gremaud, Phys. Rev. Lett. 74, 5092 (1995).

[8] P. J. Holmes, J. Sound Vib. 84, 173 (1982).

[9] A. C. J. Luo and R. P. S. Han, Nonlinear Dyn. 10, 1 (1996).
[10] R. M. Everson, Physica D 19, 355 (1986).

[11] N. B. Tufillaro, T. M. Mello, Y. M. Choi, and A. M. Albano, J. Phys. (Paris) 47, 1477 (1986).

[12] Z. J. Kowalik, M. Franaszek, and P. Pieranski, Phys. Rev. A 37, 4016 (1988).

[13] A. Mehta and J. M. Luck, Phys. Rev. Lett. 65, 393 (1990).

[14] S. Warr and J. M. Huntley, Phys. Rev. E 52, 5596 (1995).

[15] J.-C. Geminard and C. Laroche, Phys. Rev. E 68, 031305 (2003).

[16] S. Giusepponi, F. Marchesoni, and M. Borromeo, Physica A 351, 142 (2005)

[17] C. N. Bapat and S. Sankar, J. Sound Vib. 108, 99 (1986).

[18] S. Luding, E. Clément, A. Blumen, J. Rajchenbach, and J. Duran, Phys. Rev. E 49, 1634 (1994). 